\title{
SOME BASIC RESULTS FOR PROPER FREE $G$-MANIFOLDS, WHERE $G$ IS A DISCRETE GROUP
}

\author{
MARJA KANKAANRINTA \\ Department of Mathematics, PO Box 4 (Yliopistonkatu 5), FIN-00014, \\ University of Helsinki, Finland (mkankaan@cc.helsinki.fi)
}

(Received 15 May 2000)

\begin{abstract}
Let $G$ be a countable discrete group and let $M$ be a proper free $C^{r} G$-manifold and $N$ a $C^{r} G$-manifold, where $1 \leqslant r \leqslant \omega$. We prove that if $G$ acts properly and freely also on $N$ and if $\operatorname{dim}(N) \geqslant 2 \operatorname{dim}(M)$, then equivariant immersions form an open dense subset in the space $C_{G}^{r}(M, N)$ of all equivariant $C^{r}$ maps from $M$ to $N$. The space $C_{G}^{r}(M, N)$ is equipped with a topology, which coincides with the Whitney $C^{r}$ topology if $G$ is finite and is suited to studying equivariant maps. We also prove an equivariant version of Thom's transversality theorem and show that $C_{G}^{\omega}(M, N)$ is dense in $C_{G}^{r}(M, N)$, for $1 \leqslant r \leqslant \infty$.
\end{abstract}

Keywords: immersion; transverse; real analytic; dense; equivariant

AMS 2000 Mathematics subject classification: Primary 57S20

\section{Introduction}

Let $G$ be a Lie group and let $M$ and $N$ be proper $C^{r} G$-manifolds, $1 \leqslant r \leqslant \omega$ (as usual, $C^{\omega}$ denotes real analytic). We denote the space of all $C^{r} G$-maps from $M$ to $N$ by $C_{G}^{r}(M, N)$ and equip it with the strong-weak $C^{r}$ topology defined in [5], which is well suited to studying group actions. For compact $G$, this topology coincides with the Whitney $C^{r}$ topology.

Let $\operatorname{Imm}_{G}^{r}(M, N)$ and $\operatorname{Prop}_{G}^{r}(M, N)$ denote the sets of $C^{r} G$-equivariant immersions and $C^{r} G$-equivariant proper maps from $M$ to $N$, respectively. We prove the following equivariant version of Whitney's immersion theorem.

Theorem 1.1. Let $G$ be a countable discrete group and let $M$ and $N$ be proper free $C^{r} G$-manifolds, where $1 \leqslant r \leqslant \omega$ and $\operatorname{dim}(N) \geqslant 2 \operatorname{dim}(M)$. Then

(1) $\operatorname{Imm}_{G}^{r}(M, N)$ is open and dense in $C_{G}^{r}(M, N)$, and

(2) $\operatorname{Imm}_{G}^{r}(M, N) \cap \operatorname{Prop}_{G}^{r}(M, N)$ is open and dense in $\operatorname{Prop}_{G}^{r}(M, N)$.

We also prove an equivariant version of Thom's transversality theorem.

Theorem 1.2. Let $G$ be a countable discrete group and let $M$ be a proper free $C^{r} G$ manifold and $N$ a $C^{r} G$-manifold, where $1 \leqslant r \leqslant \omega$. Let $N^{\prime}$ be a closed $C^{r} G$-submanifold 
of $N$, and $L$ a closed $G$-invariant subset of $M$. Then the set of $C^{r} G$-maps from $M$ to $N$ which are transverse to $N^{\prime}$ along $L$ is open and dense in $C_{G}^{r}(M, N)$.

Theorem 1.2 does not always hold if the action of $G$ on $M$ is not free (see $[\mathbf{1}, \S 2]$ or $[\mathbf{2}, \S 2])$. In both cases, easy counterexamples are constructed, where a finite group acts non-freely.

Finally, we obtain the following density result for real analytic $G$-equivariant maps.

Theorem 1.3. Let $G$ be a countable discrete group and let $M$ be a proper free $C^{\omega}$ $G$-manifold and $N$ a $C^{\omega} G$-manifold. Then $C_{G}^{\omega}(M, N)$ is dense in $C_{G}^{r}(M, N)$, where $1 \leqslant r \leqslant \infty$.

Since the set of all $G$-equivariant diffeomorphisms is open in $C_{G}^{r}(M, N)[\mathbf{5}$, Theorem 7.5], we obtain the following corollary.

Corollary 1.4. Let $G$ be a countable discrete group and let $M$ and $N$ be proper free $C^{\omega} G$-manifolds. If $M$ and $N$ are $C^{r} G$-diffeomorphic, where $1 \leqslant r \leqslant \infty$, then they are $C^{\omega} G$-diffeomorphic.

In $\left[6\right.$, Theorem II] it was proved that $C_{G}^{\omega}(M, N)$ is dense in $C_{G}^{r}(M, N)$, where $1 \leqslant r \leqslant$ $\infty$, if $G$ is a closed subgroup of a virtually connected Lie group and $M$ and $N$ are proper $C^{\omega} G$-manifolds. Using the same simple idea as in the proofs of Theorems 1.2 and 1.3, one can also drop the assumption there that the action on $N$ is proper.

\section{Proofs of the theorems}

Throughout the paper, let $G$ be a countable discrete group. We call $M$ a proper free $C^{r}$ $G$-manifold, where $1 \leqslant r \leqslant \omega$, if the action $G \times M \rightarrow M$ is properly discontinuous, free and $C^{r}$ differentiable. Then the map $G \times M \rightarrow M \times M,(g, x) \mapsto(x, g x)$, is a proper map, i.e. the inverse image of every compact set is compact for it. By [9, Corollary I 3.24], the orbit map $\pi_{M}: M \rightarrow M / G$ is a covering, i.e. a locally trivial map with fibre $G$. Unless otherwise stated, $M$ and $N$ will be proper free $C^{r} G$-manifolds, where $1 \leqslant r \leqslant \omega$. All manifolds are assumed to be finite dimensional, second countable and without boundary.

As mentioned in $\S 1$, the topology in the set $C_{G}^{r}(M, N), 1 \leqslant r \leqslant \omega$, of all $C^{r} G$-maps from $M$ to $N$ is the strong-weak $C^{r}$ topology defined in $[\mathbf{5}, \S \S 1,4]$. This topology depends on the action of $G$ and coincides with the Whitney $C^{r}$ topology (see, for example, $[4$, Chapter 2]) if $G$ is finite. In particular, whenever we consider spaces of maps between manifolds without a group action, the topology will be the Whitney $C^{r}$ topology. In the strong-weak $C^{r}$ topology the basic neighbourhoods can be defined in the same way as in the Whitney $C^{r}$ topology, but by using only families of charts in $M$ whose images in the orbit space $M / G$ form a locally finite family. For the notation of basic neighbourhoods etc., see [5].

Lemma 2.1. Let $N^{\prime}$ be a closed $C^{r} G$-submanifold of $N$ and let $x \in M$. Let $f: M \rightarrow N$ be a $G$-equivariant map and $\tilde{f}: M / G \rightarrow N / G$ the map induced by $f$. Then

(1) $f$ is $C^{k}$ differentiable, if and only if $\tilde{f}$ is $C^{k}$ differentiable, $1 \leqslant k \leqslant r$; 
(2) $f$ is an immersion, if and only if $\tilde{f}$ is an immersion;

(3) $f$ is a submersion, if and only if $\tilde{f}$ is a submersion;

(4) $f$ is proper, if and only if $\tilde{f}$ is proper;

(5) $f$ is transverse to $N^{\prime}$ at $x$, if and only if $\tilde{f}$ is transverse to $N^{\prime} / G$ at $\pi_{M}(x)$;

(6) $f$ is a (closed) $C^{k}$ embedding, if and only if $\tilde{f}$ is a (closed) $C^{k}$ embedding; and

(7) $f$ is a $C^{k}$ diffeomorphism, if and only if $\tilde{f}$ is a $C^{k}$ diffeomorphism.

Proof. The first three claims follow at once from the fact that the orbit maps are coverings. The fourth claim follows from [5, Lemmas 3.7 and 3.9]. The last three claims are easy to verify.

Let $f_{0}, f_{1}: M \rightarrow N$ be $C^{r}$ maps. By a $C^{r}$ homotopy between $f_{0}$ and $f_{1}$ we mean a homotopy $M \times I \rightarrow N$ between $f_{0}$ and $f_{1}$ which can be extended to be $C^{r}$ on $M \times J$, where $J$ is some open interval containing the unit interval $I$.

The following version of the covering homotopy theorem of Palais (see [7, Theorem 2.4.1]) holds for properly discontinuous free $C^{r}$ actions. Although first proved for actions of compact groups, the covering homotopy theorem holds for proper actions as well, as pointed out by Palais in $[\mathbf{8}, \S 4.5]$. The lift is of class $C^{k}$ by part (1) of Lemma 2.1.

Theorem 2.2. Let $G$ be a countable discrete group and let $M$ and $N$ be proper free $C^{r} G$-manifolds, $1 \leqslant r \leqslant \omega$. Let $f: M \rightarrow N$ be a $C^{k} G$-map, $0 \leqslant k \leqslant r$. If $\tilde{H}: M / G \times I \rightarrow N / G$ is any $C^{k}$ homotopy of the induced map $\tilde{f}$, then there exists a $C^{k}$ G-homotopy $H: M \times I \rightarrow N$ of $f$ with induced map $\tilde{H}$.

Lemma 2.3. Let $M$ and $N$ be $C^{r}$ manifolds, $1 \leqslant r \leqslant \omega$. Let $f: M \rightarrow N$ be a $C^{r}$ map and let $\mathcal{N}$ be a neighbourhood of $f$ in $C^{r}(M, N)$. Then $f$ has a neighbourhood $\mathcal{M}$ in $C^{r}(M, N)$ such that every $h \in \mathcal{M}$ is homotopic to $f$ by some $C^{r}$ homotopy $H: M \times I \rightarrow N$ and $H_{t} \in \mathcal{N}$ for every $t \in I$.

Proof. We can assume that $\mathcal{N}$ is a basic neighbourhood, i.e. of form

$$
\bigcap_{i \in \Lambda} \mathcal{N}^{r}\left(f ; K_{i},\left(U_{i}, \varphi_{i}\right),\left(V_{i}, \psi_{i}\right), \varepsilon_{i}\right)
$$

(see [5]). Let $J$ be some bounded open interval containing $I$ and let $H_{f}: M \times J \rightarrow N$ be the constant extension of the constant homotopy induced by $f$. Then

$$
\tilde{\mathcal{N}}=\bigcap_{i \in \Lambda} \mathcal{N}^{r}\left(H_{f} ; K_{i} \times I,\left(U_{i} \times J, \varphi_{i} \times \mathrm{id}\right),\left(V_{i}, \psi_{i}\right), \varepsilon_{i}\right)
$$

is a neighbourhood of $H_{f}$. By the embedding theorems of Whitney $(1 \leqslant r \leqslant \infty)$ and Grauert $(r=\omega)$, there exists a closed $C^{r}$ embedding $e: N \rightarrow \mathbb{R}^{p}$, for some $p$. Let $r: W \rightarrow$ $e(N)$ be a $C^{r}$ tubular neighbourhood of $e(N)$. Let $\mathcal{W} \subset C^{r}(M, N)$ be a neighbourhood of $f$ such that if $h \in \mathcal{W}$, then

$$
\text { te } \circ f(x)+(1-t) e \circ h(x) \in W,
$$


for every $t \in J$ and for every $x \in M$. Then the mapping

$$
\begin{gathered}
A: \mathcal{W} \rightarrow C^{r}(M \times J, N), \\
A(h)(x, t)=e^{-1} \circ r \circ(t e \circ f(x)+(1-t) e \circ h(x)),
\end{gathered}
$$

is continuous in the Whitney $C^{r}$ topology and $A(f)=H_{f}$. Thus $f$ has a neighbourhood $\mathcal{M}$ such that $A(\mathcal{M}) \subset \tilde{\mathcal{N}}$. Therefore $A(h)_{t} \in \mathcal{N}$, for every $h \in \mathcal{M}$ and for every $t \in I$.

The following theorem will be crucial in proving Theorems 1.1, 1.2 and 1.3.

Theorem 2.4. The map

$$
\tau: C_{G}^{r}(M, N) \rightarrow C^{r}(M / G, N / G),
$$

taking $f$ to the induced map $\tilde{f}$, is open and continuous.

Proof. We begin by proving the continuity. Let $f \in C_{G}^{r}(M, N)$ and let

$$
\tilde{\mathcal{N}}=\bigcap_{i \in \Lambda} \mathcal{N}^{r}\left(\tilde{f} ; \tilde{K}_{i},\left(\tilde{U}_{i}, \tilde{\varphi}_{i}\right),\left(\tilde{V}_{i}, \tilde{\psi}_{i}\right), \varepsilon_{i}\right)
$$

be a basic neighbourhood of $\tilde{f}$ such that $\tilde{K}_{i}$ is connected and the diagrams
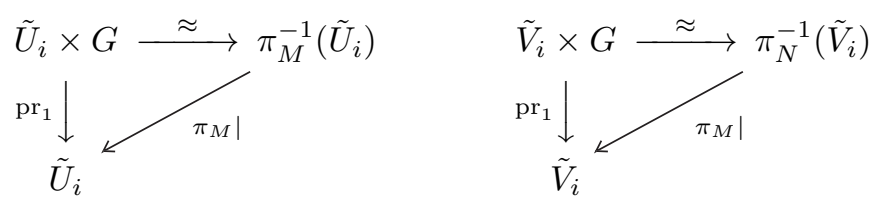

are commutative for every $i$. The restrictions $\pi_{M} \mid: U_{i} \rightarrow \tilde{U}_{i}$ and $\pi_{N} \mid: V_{i} \rightarrow \tilde{V}_{i}$ are diffeomorphisms, for some charts $U_{i}$ of $M$ and $V_{i}$ of $N$, respectively, where $G U_{i}=\pi_{M}^{-1}\left(\tilde{U}_{i}\right)$, $G V_{i}=\pi_{N}^{-1}\left(\tilde{V}_{i}\right)$ and $f\left(\pi_{M}^{-1}\left(\tilde{K}_{i}\right) \cap U_{i}\right) \subset V_{i}$. Then

$$
\mathcal{N}=\bigcap_{i \in \Lambda} \mathcal{N}^{r}\left(f ; \pi_{M}^{-1}\left(\tilde{K}_{i}\right) \cap U_{i},\left(U_{i}, \tilde{\varphi}_{i} \circ \pi_{M} \mid\right),\left(V_{i}, \tilde{\psi}_{i} \circ \pi_{N} \mid\right), \varepsilon_{i}\right)
$$

is a basic neighbourhood of $f$ in $C_{G}^{r}(M, N)$ and $\tau(\mathcal{N}) \subset \tilde{\mathcal{N}}$. Consequently, $\tau$ is continuous at $f$. Since $f$ was arbitrary, it follows that $\tau$ is continuous.

It remains to prove that $\tau$ is open. It suffices to show that $\tau$ maps every basic neighbourhood onto an open set in $C^{r}(M / G, N / G)$. Let $f, \tilde{f}, \mathcal{N}$ and $\tilde{\mathcal{N}}$ be as above. By Lemma 2.3, $\tilde{f}$ has a neighbourhood $\tilde{\mathcal{M}}$ such that every $\tilde{h} \in \tilde{\mathcal{M}}$ is homotopic to $\tilde{f}$ by some $C^{r}$ homotopy $\tilde{H}: M / G \times I \rightarrow N / G$ and $\tilde{H}_{t} \in \tilde{\mathcal{N}}$ for every $t \in I$. Since the sets of form $\mathcal{N} \cap \tau^{-1}(\tilde{\mathcal{M}})$ form a neighbourhood basis at $f$, it is enough to show that $\tau$ maps $\mathcal{N} \cap \tau^{-1}(\tilde{\mathcal{M}})$ onto an open set in $C^{r}(M / G, N / G)$. We will show that $\tau\left(\mathcal{N} \cap \tau^{-1}(\tilde{\mathcal{M}})\right)=\tilde{\mathcal{M}}$.

Assume $\tilde{h} \in \tilde{\mathcal{M}}$ and let $\tilde{H}$ be a $C^{r}$ homotopy between $\tilde{f}$ and $\tilde{h}$ with $\tilde{H}_{t} \in \tilde{\mathcal{N}}$ for every $t \in I$. By Theorem 2.2, $\tilde{H}$ has a $C^{r} G$-equivariant lift $H: M \times I \rightarrow N$ such that $H_{0}=f$. It suffices to show that $H_{1} \in \mathcal{N}$. Let $x \in \pi_{M}^{-1}\left(\tilde{K}_{i}\right) \cap U_{i}$, for some $i \in \Lambda$. Then $H_{t}(x) \in G V_{i}$, for every $t \in I$. Since $f(x) \in V_{i}$ and $V_{i} \cap g V_{i}=\emptyset$ unless $g$ equals the identity element, 
it follows that $H(\{x\} \times I) \subset V_{i}$. In particular, $H_{1}(x) \in V_{i}$. Since this holds for every $x \in \pi_{M}^{-1}\left(\tilde{K}_{i}\right) \cap U_{i}$ and for every $i \in \Lambda$ and also the required inequalities for the norms of the differences of the partial derivatives of $f$ and $H_{1}$ clearly hold, it follows that $H_{1} \in \mathcal{N}$.

Proof of Theorem 1.1. By [4, Theorems 2.1.1 and 2.1.5], $\operatorname{Imm}^{r}(M / G, N / G)$ and $\operatorname{Prop}^{r}(M / G, N / G)$ are open in $C^{r}(M / G, N / G)$. Thus the openness claims follow by using parts (2) and (4) of Lemma 2.1 and the fact that $\tau$ is continuous. By [4, Theorem 2.2.12], $\operatorname{Imm}^{r}(M / G, N / G)$ is dense in $C^{r}(M / G, N / G)$. The density claims follow by using parts (2) and (4) of Lemma 2.1 and the fact that $\tau$ is open.

Notice that for the openness results one in fact does not need to assume that $G$ is a discrete group acting freely and properly discontinuously. The strong-weak topology for $C_{G}^{r}(M, N)$ is defined when $G$ is any Lie group and, by [5, Propositions 6.1 and 6.4], the sets $\operatorname{Imm}_{G}^{r}(M, N)$ and $\operatorname{Prop}_{G}^{r}(M, N)$ are open in $C_{G}^{r}(M, N)$, assuming that $G$ acts properly on $M$ and $N$.

Proof of Theorem 1.2. If the action of $G$ on $N$ is free and properly discontinuous, then the claim follows from Thom's transversality theorem (see, for example, $[4$, Theorem 3.2.1]), part (5) of Lemma 2.1 and Theorem 2.4. The proof is similar to that of Theorem 1.1.

Assume then that the $C^{r}$ action of $G$ on $N$ is arbitrary. This case can be reduced to the case of a proper free action. Namely, the diagonal action of $G$ on $M \times N$ is proper and free and of class $C^{r}$. Moreover, a $C^{r} G$-map $f: M \rightarrow N$ is transverse to $N^{\prime}$ along $L$, if and only if for every $h \in C_{G}^{r}(M, M),(h, f): M \rightarrow M \times N$ is transverse to $M \times N^{\prime}$ along $L$. Both the density and openness results follow easily by using Proposition 4.6 in [5], according to which there is a canonical homeomorphism

$$
C_{G}^{r}(M, M \times N) \approx C_{G}^{r}(M, M) \times C_{G}^{r}(M, N) .
$$

Proof of Theorem 1.3. If $G$ acts freely and properly on $N$, then the claim follows as the proofs of Theorems 1.1 and 1.2, by using Whitney's approximation theorem, which implies that $C^{\omega}(M / G, N / G)$ is dense in $C^{r}(M / G, N / G)$, and part (1) of Lemma 2.1. The proof of the case of an arbitrary $C^{\omega}$ action of $G$ on $N$ can be reduced to the case of a proper free action, as in the proof of Theorem 1.2.

Remark 2.5. In [4], Whitney's immersion theorem and Thom's transversality theorem are only stated for the cases $1 \leqslant r \leqslant \infty$. However, the $C^{\omega}$ case follows easily from Whitney's approximation theorem, according to which $C^{\omega}$ maps form a dense set in $C^{\infty}(M, N)$ when $M$ and $N$ are $C^{\omega}$ manifolds.

Remark 2.6. Studying ordinary transversality in the equivariant case only makes sense because the action of $G$ on $M$ is free and properly discontinuous. For smooth actions of a compact Lie group there exist the notions of general position (see [1] ) and 
$G$-transversality (see $[\mathbf{2}]$ ). These concepts are equivalent by $[\mathbf{3}]$ and agree with ordinary transversality if $G$ is a finite group acting freely. Notice that if one tries to generalize the results in $[\mathbf{1}]$ and $[\mathbf{2}]$ to the case of proper actions of non-compact Lie groups, one should not work with the Whitney $C^{r}$ topology in $C_{G}^{r}(M, N)$, which is discrete [5, Proposition 4.7], but with the strong-weak $C^{r}$ topology.

Acknowledgements. The author was supported by the Jenny and Antti Wihuri foundation.

\section{References}

1. E. Bierstone, General position of equivariant maps, Trans. Am. Math. Soc. 234 (1977), 447-466.

2. M. Field, Transversality in G-manifolds, Trans. Am. Math. Soc. 231 (1977), 429-450.

3. M. Field, Stratifications of equivariant varieties, Bull. Aust. Math. Soc. 16 (1977), 279295.

4. M. Hirsch, Differential topology (Springer, 1976).

5. S. Illman and M. KankaAnRinta, A new topology for the set $C^{\infty, G}(M, N)$ of $G$ equivariant smooth maps, Math. Annln 316 (2000), 139-168.

6. S. Illman and M. KankaAnRinta, Some basic results for real analytic proper $G$ manifolds, Math. Annln 316 (2000), 169-183.

7. R. S. PALAis, The classification of G-spaces, Memoirs of the American Mathematical Society, vol. 36 (1960).

8. R. S. Palais, On the existence of slices for actions of non-compact Lie groups, Ann. Math. (2) 73 (1961), 295-323.

9. T. том DiECK, Transformation groups (Walter de Gruyter, Berlin, 1987). 